06

\title{
Комплексные исследования кинетики индуцированного фазового перехода в [001], [011] и [111]-ориентированных монокристаллических релаксорных твердых растворах
}

\author{
(С) Л.С. Камзина, Л.А. Кулакова \\ Физико-технический институт им. А.Ф. Иофффе РАН, \\ Санкт-Петербург, Россия \\ E-mail: kamzin@mail.ioffe.ru
}

(Поступила в Редакцию 23 ноября 2017 г.

В окончательной редакции 5 декабря 2017 г.)

При комнатной температуре исследована кинетика индуцированного фазового перехода в монокристаллических релаксорных твердых растворах $33 \mathrm{PbIn}_{1 / 2} \mathrm{Nb}_{1 / 2} \mathrm{O}_{3}-35 \mathrm{PbMg}_{1 / 3} \mathrm{Nb}_{2 / 3} \mathrm{O}_{3}-32 \mathrm{PbTiO}_{3}$ и $\mathrm{PbMg}_{1 / 3} \mathrm{Nb}_{2 / 3} \mathrm{O}_{3}-29 \mathrm{PbTiO}_{3}$ при приложении электрического поля вдоль трех направлений [001], [011] и [111]. Проведены совместные измерения оптического пропускания на разных длинах волн, диэлектрических свойств, а также скорости и затухания звука. Обнаружено, что даже в небольших полях $(E \sim 2 \mathrm{kV} / \mathrm{cm})$, приложенных в направлениях [011] и [111], максимальные изменения всех трех характеристик, а также совпадение временных зависимостей оптического пропускания на разных длинах волн света происходят в одном и том временном интервале. Установлено, что в том же интервале полей, направленных вдоль [001], аномалии оптических свойств не совпадают по времени с аномалиями акустических и диэлектрических свойств. Полученные результаты объясняются индуцированием монодоменной и многодоменной фаз.

DOI: 10.21883/FTT.2018.05.45793.297

\section{1. Введение}

Необычные свойства релаксоров связаны с присутствием в параэлектрической фазе локальных пространственно ориентированных сегнетоэлектрических наноразмерных областей ромбоэдрической симметрии (полярные нанообласти PNR). Эти области возникают при температуре Бернса $\left(T_{B} \sim 620-650 \mathrm{~K}\right)$, значительно превышающей температуру максимума диэлектрической проницаемости $(\varepsilon)$, и имеют размеры 2-10 nm. При понижении температуры ниже температуры максимума $\varepsilon$ PNR начинают увеличиваться в размеpax до температуры замерзания $T_{f}$ (или температура Фогеля-Фулчера). Ниже $T_{f}$ возникает неэргодическое стеклоподобное состояние, в котором размеры PNR остаются неизменными $(10-30 \mathrm{~nm})$. В таком замороженном релаксорном состоянии PNR приобретают слабую кинетику, но не образуют дальнего порядка. Они ведут себя как большие дипольные моменты, совершающие тепловые флуктуации между эквивалентными потенциальными ямами, и наблюдаемые свойства релаксоров связаны с переориентацией этих диполей. Широкий спектр времен релаксации спонтанной поляризации является основной чертой неравновесного состояния стекольной системы. Он связан как с распределением величин потенциальных барьеров, разделяющих состояния с различной ориентацией, так и с разными размерами полярных областей. Свойства кристалла в таком неравновесном состоянии должны зависеть от времени. Макродоменное состояние с дальним порядком возникает только в электрическом поле.
При исследовании нейтронов, а также диэлектрических свойств классического релаксора $\mathrm{PbMg}_{1 / 3} \mathrm{Nb}_{2 / 3} \mathrm{O}_{3}$ $(\mathrm{PMN})$ и ряда релаксорных твердых растворов на его основе $\mathrm{PbMg}_{1 / 3} \mathrm{Nb}_{2 / 3} \mathrm{O}_{3}-6 \mathrm{PbTiO}_{3} \quad(\mathrm{PMN}-6 \mathrm{PT})$ и $\mathrm{PbMg}_{1 / 3} \mathrm{Nb}_{2 / 3} \mathrm{O}_{3}-12 \mathrm{PbTiO}_{3}$ (PMN-12PT), авторы [1-5] обнаружили, что при приложении электрического поля в стеклоподобной фазе фазовый переход в макродоменное состояние проходит в два этапа.

Во время первого этапа кинетика определяется приблизительно логарифмическим ростом поляризации в стекольной фазе, при этом формируются малые области ромбоэдрической фазы. На втором этапе происходит укрупнение и слияние полярных областей, что приводит к индуцированию сегнетоэлектрического фазового перехода и образованию дальнего порядка. В наших последующих работах [6-10] мы продолжили изучение кинетики индуцированного фазового перехода, расширив класс веществ и используя другие методики измерений, а именно оптические и акустические. Исследовались двойные $\mathrm{PMN}-x$ PT и тройные твердые растворы $\quad \mathrm{PbIn}_{1 / 2} \mathrm{Nb}_{1 / 2} \mathrm{O}_{3}-\mathrm{PbMg}_{1 / 3} \mathrm{Nb}_{2 / 3} \mathrm{O}_{3}-x \mathrm{PbTiO}_{3}$ $(\mathrm{PIN}-\mathrm{PMN}-x \mathrm{PT})$ с разной величиной $x$, лежащие как вдали от морфотропной фазовой границы (МФГ), так и вблизи нее. Мы показали, что при приложении электрического поля ниже температуры морфотропного фазового перехода (МФП), фазовый переход в макродоменное состояние проходит в два этапа даже в соединениях, лежащих вблизи МФГ, что свидетельствует о существовании в них небольшой доли релаксорной фазы. В нашей работе [10] мы проводили совместные наблюдения изменений во времени оптических и акустиче- 
ских свойств в электрическом поле, приложенном вдоль направления [001], в кристаллах 20PIN-55PMN-25PT, 33PIN-35PMN-32PT (PT32) и PMN-29PT (PT29). Было обнаружено, что во всех исследованных кристаллах резкое уменьшение оптического пропускания в видимой области спектра наблюдается раньше, чем аномалии акустических свойств, в то время как в инфракрасном диапазоне оно происходит в более широком временном интервале и наблюдается даже после аномалий акустических параметров. Из полученных данных был сделан вывод, что зависимость пропускания от длины волны света связана только с увеличением размеров нанообластей в процессе прохождения фазового перехода. На основании этих результатов нами было высказано предположение, что используемые в работе электрические поля, приложенные в направлении [001], приводят не к полной (монодоменное состояние), а только к частичной поляризации образца, и дальний порядок возникает в части объема кристалла. Как следует из рентгеновских и доменных исследований $[11,12]$, поле, приложенное вдоль направления [001], индуцирует в этих соединениях переход в моноклинную фазу, а вдоль [011] — в орторомбическую.

Каким образом проявляется кинетика образования зародышей новой фазы в электрических полях, приложенных в других направлениях, было не известно. Кроме того, не исследовалась динамика изменений со временем диэлектрических свойств в данных кристаллах, а также связь этих изменений с изменениями оптических и акустических свойств при совместных измерениях всех трех величин. В настоящей работе проведены одновременные измерения изменений оптических (для двух длин волн), акустических и диэлектрических свойств в кристаллах PT29 и РТ32 в электрических полях, приложенных в направлениях [001], [011] и [111], с целью получения более полной информации о поляризации образцов и механизмaх, определяющих изменения этих свойств в различных геометриях приложения электрического поля.

\section{2. Образцы и методика эксперимента}

Кристаллы выращивали прямо из расплава модифицированным методом Бриджмена [13]. Были получены высококачественные монокристаллы 33PIN-35PMN32PT (РТ32) и PMN-29PТ (РТ29). Кристаллы РТ32 ориентировались и затем разрезались вдоль плоскостей (011), а кристаллы РТ 29 вдоль плоскостей (001) и (111). Как следует из рентгеновских и нейтронных исследований, симметрия всех исследуемых в работе образцов при комнатной температуре в отсутствие электрического поля в целом кубическая. Эта симметрия является усредненной и играет роль матрицы, в которую внедрены нанообласти разной локальной симметрии. Основная часть таких областей имеет ромбоэдрическое искажение с поляризацией вдоль [111]. Наряду с ними присутствуют отдельные PNR моноклинной, орторомбической и тетрагональной фаз [14].

Для оптических измерений использовались $\mathrm{He}-\mathrm{Ne}-$ лазер $(\lambda=0.63 \mu \mathrm{m}$, непрерывный режим) и полупроводниковый лазер $(\lambda=1.3 \mu \mathrm{m}$, импульсный режим). Измерения проводили при комнатной температуре. Электрическое поле прикладывалось в направлениях [001], [011] и [111] в стеклоподобной релаксорной фазе и совпадало по направлению с распространением звуковой волны. Пропускание света измеряли в перпендикулярном направлении. При непрерывном режиме работы лазера $(\lambda=0.63 \mu \mathrm{m})$ импульс света формировался дисковым прерывателем. Им же вырабатывался импульс синхронизации запуска ВЧ генератора, вырабатывающего акустический импульс. В инфракрасном диапазоне $(\lambda=1.3 \mu \mathrm{m})$ синхронизация оптических и акустических импульсов осуществлялась сигналом ВЧ генератора. Для измерения акустических свойств кристаллов использовали эхо-импульсную методику [15]. Ультразвуковые импульсы частотой $20 \mathrm{MHz}$ и длительностью до $0.5 \mu$ s возбуждались с помощью генератора сигналов AFG фирмы INSTEK резонансными пластинками из пьезоэлектрической керамики, приклеиваемыми к образцу. Широкополосный (полоса $200 \mathrm{MHz}$ ) осциллограф (фирмы Tectronix) и камера Sony Cybershot использовались для фиксированной и непрерывной записи поведения оптических и акустических импульсов, а также изменения диэлектрической проницаемости $(\varepsilon)$, которая регистрировалась измерителем иммитанса E 7-15 на частоте $1 \mathrm{kHz}$.

Перед каждым измерением образцы отжигали в течение часа при $\sim 500 \mathrm{~K}$. После отжига образцы охлаждали без поля до комнатной температуры. Электрическое поле с напряженностью, достаточной для индуцирования фазового перехода в сегнетоэлектрическое состояние, прикладывалось через 40 min после отжига кристалла.

\section{3. Экспериментальные результаты}

На рис. $1, a, b$ представлены временные зависимости изменений оптического пропускания на разных длинах волн, одновременно измеренные с ним скорости и затухания звука при фиксированной частоте, а также изменения диэлектрической проницаемости в кристалле РТ29 в электрическом поле, приложенном вдоль [001]. Как видно из рис. 1, $a$, перед началом измерений в отсутствие поля, оптическое пропускание максимально на обеих длинах волн. Из этого следует, что в этом кристалле в отсутствие поля в стеклоподобной релаксорной фазе все сосуществующие нанообласти имеют размеры меньше длины волны света, как для $0.63 \mu \mathrm{m}$, так и для $1.3 \mu \mathrm{m}$. При приложении электрического поля со временем оптическое пропускание в видимой области на длине $0.63 \mu \mathrm{m}$ (кривая 1 ) резко уменьшается в отличие от его поведения в инфракрасной области $(1.3 \mu \mathrm{m})$ (кривая 2). Следует заметить, что изменение 

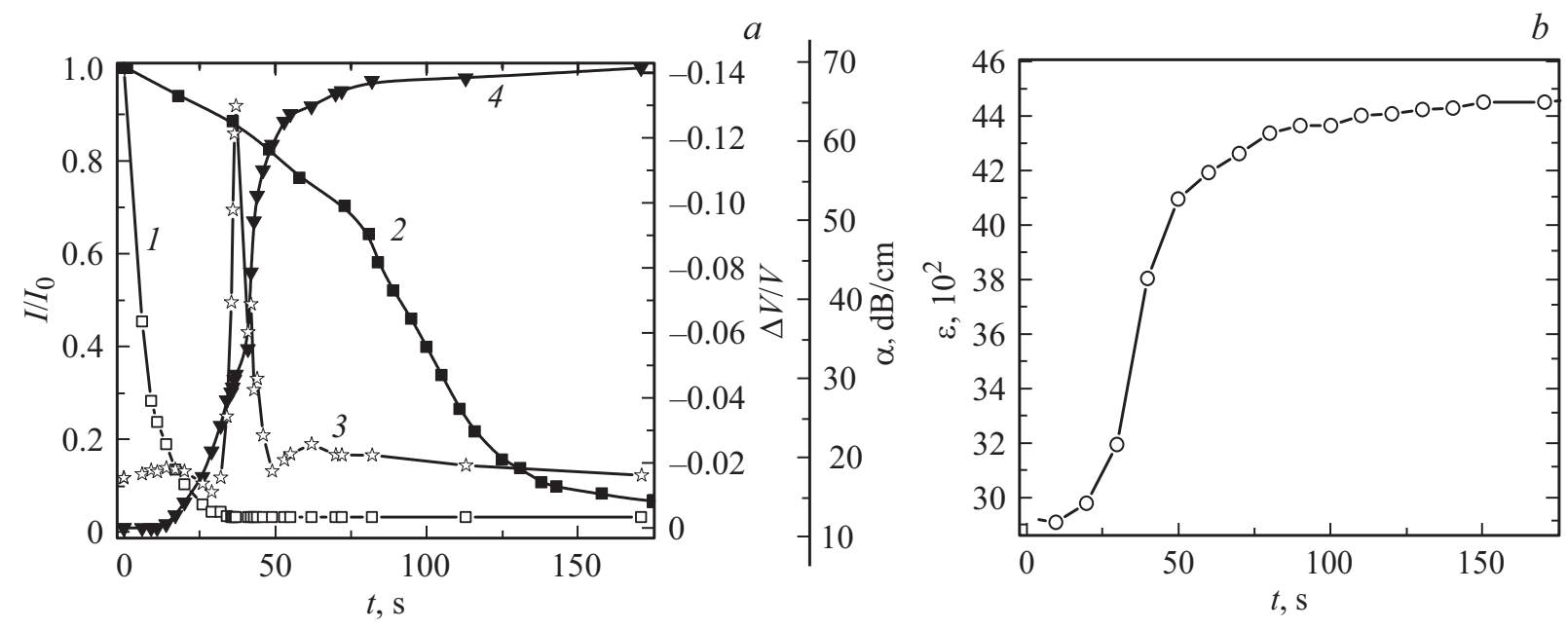

Рис. 1. Временные зависимости основных характеристик кристалла РТ29 в электрическом поле $E=1.23 \mathrm{kV} / \mathrm{cm}$, приложенном вдоль $[001],(a)-$ оптического пропускания $(1,2)$ при разных длинах волн света, $\lambda, \mu \mathrm{m}: 1-0.63,2-1.3$; затухания $(3)$ и скорости звука (4); (b) - диэлектрической проницаемости.

оптического пропускания происходит в другом временном интервале, чем изменение акустических свойств (кривые 3,4). Наиболее заметное уменьшение оптического пропускания на длине волны $1.3 \mu \mathrm{m}$, как видно из рис. 1, $a$ (кривая 2), наблюдается после аномалий акустических свойств (кривые 3,4 ). Ранее в работе [9] мы показали, что положение акустических аномалий (резкий рост скорости и максимум затухания) не зависит от частоты, а, следовательно, и от длины волны звука. Из этого можно сделать вывод, что наблюдаемые аномалии не являются размерным эффектом, а возникают вследствие изменения упругих свойств в объеме, которые характерны для фазового перехода. В области фазового перехода в узком интервале времени эти величины резко меняются. Наблюдается большой пик затухания, что является естественным в условиях перестройки кристаллической структуры, и резкое возрастание скорости звука из-за увеличения жесткости решетки, вызванного большим пьезоэлектрическим эффектом в сегнетоэлектрической фазе. Несовпадение по времени максимального изменения оптического пропускания с временными аномалиями акустических свойств свидетельствуют о том, что электрическое поле, используемое в данной работе, не приводит к полной монодоменизации образца [10]. Для полной поляризации всего образца и перехода в монодоменную моноклинную фазу требуется больше времени или бо́льшие электрические поля.

В отличие от оптического пропускания, максимальное изменение диэлектрической проницаемости (рис. $1, b$ ) совпадает по времени с акустическими аномалиями. Хорошо известно, что диэлектрические свойства кристалла так же, как и акустические, очень чувствительны к изменению структуры. Приложение поля вдоль направления [001] увеличивает со временем величину $\varepsilon$. Это может быть связано с тем, что при приложении поля вдоль [001] происходит поворот плоскости поляризации ромбоэдрических областей от направления [111] к направлению [001] через промежуточную моноклинную фазу. Как показывают результаты наших измерений (рис. $1, b)$, величина $\varepsilon$ существенно возрастает в этой фазе по сравнению с $\varepsilon$ в ромбоэдрической многодоменной фазе, которая существует в отсутствие поля.

Как мы уже упоминали выше, в твердых растворах, таких как РТ29 и РТ32, расположенных вблизи морфотропной фазовой границы, помимо PNR ромбоэдрической симметрии могут существовать нанообласти другой локальной симметрии, которые могут переключаться в направлениях [001], [011] и [111] в зависимости от направления приложенного электрического поля. На рис. 2, $a$ представлены в качестве примера временные зависимости изменений оптического пропускания для разных длин волн (кривые 1,2), скорости и затухания звука при фиксированной частоте (кривые 3,4 ), a также диэлектрической проницаемости (рис. 2,b) в кристалле РТ32 в электрическом поле, приложенном вдоль [011]. Так же, как и в случае приложения поля вдоль направления [001] (рис. 1, $a, b)$, фазовый переход происходит в два этапа с временной задержкой, во время которой происходит укрупнение областей релаксорной стеклоподобной фазы и поворот областей ромбоэдрической симметрии в направлении приложения поля [011], что приводит к возникновению областей ромбической симметрии с локальной спонтанной поляризацией, направленной вдоль [011].

Поведение временных зависимостей акустических и диэлектрических аномалий в случае поля вдоль [011] (рис. $2, a, b)$ идентично, как и для рассмотренного выше случая приложения поля вдоль [001]. Следует заметить, что изменение затухания в области фазового перехода проходит так быстро, что нам не удалось зафиксировать момент максимального значения акустического затуха- 



Рис. 2. Временные зависимости основных характеристик кристалла РТ32 в электрическом поле $E=1.87 \mathrm{kV} / \mathrm{cm}$, приложенном вдоль [011], (a) - оптического пропускания $(1,2)$ при разных длинах волн света, $\lambda, \mu \mathrm{m:} 1-0.63,2-1.3$; затухания $(3)$ и скорости звука $(4) ;(b)$ - диэлектрической проницаемости.
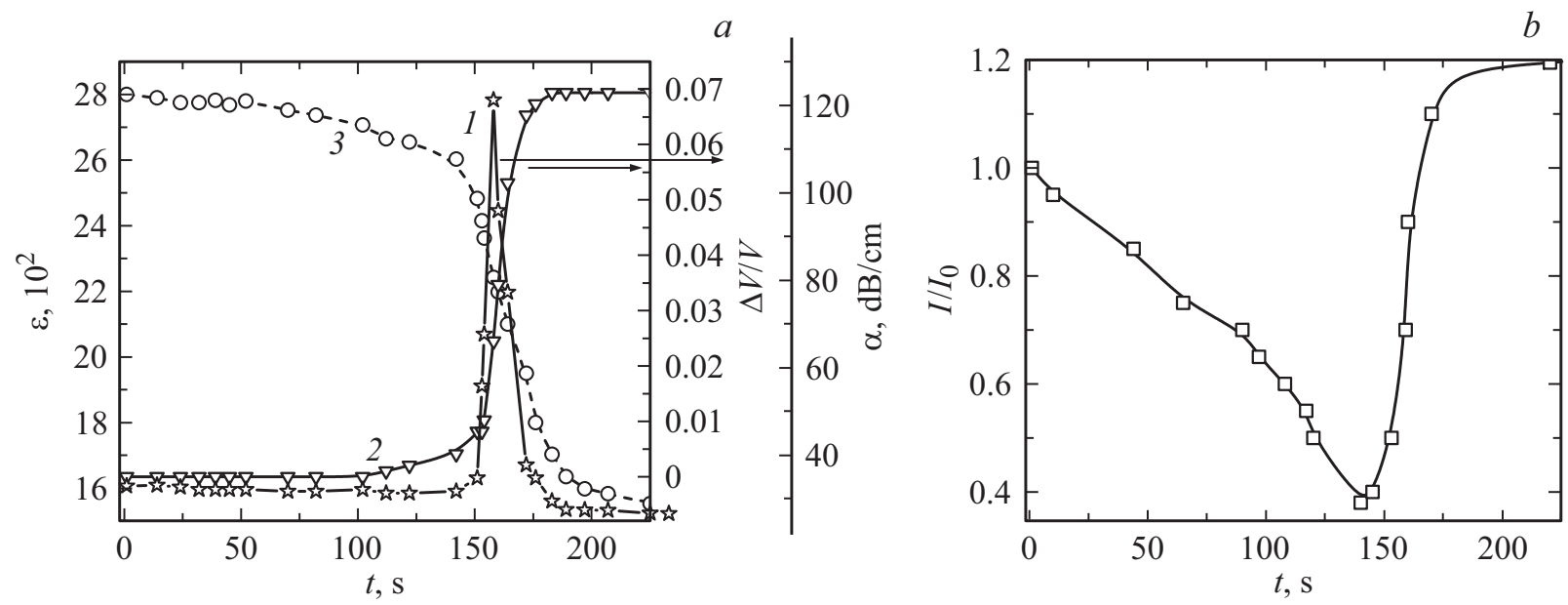

Рис. 3. Временные зависимости основных характеристик кристалла РТ29 в электрическом поле $E=1.06 \mathrm{kV} / \mathrm{cm}$, приложенном вдоль [111], (a) - затухания (1) и скорости звука (2), диэлектрической проницаемости (3); $(b)$ - оптического пропускания $\lambda=0.63 \mu \mathrm{m}$.

ния, и на рисунке виден только небольшой максимум и резкое уменьшение затухания.

Другая картина наблюдается для оптического пропускания, измеренного на двух длинах волн. Как видно из рис. 2,a (кривые 1,2) аномалии оптического пропускания, измеренные на двух длинах волн, практически совпадают друг с другом и с аномалиями акустических (кривые 3,4) и диэлектрических свойств (рис. 2,b). Пропускание сначала уменьшается, а потом увеличивается скачком. Уменьшение пропускания связано с увеличением размеров доменов, которое при переходе в орторомбическую фазу приводит к монодоменизации образца и увеличению пропускания. Возникшая при комнатной температуре орторомбическая фаза оставалась стабильной в течение длительного времени и после снятия электрического поля, т.е. в достаточно низком поле $\sim 2 \mathrm{kV} / \mathrm{cm}$ в кристалле возникла необратимая ор- торомбическая фаза. При этом переходе одновременно меняются и оптическое пропускание, и скорость звука. Эти данные согласуются с результатами работы [16] по исследованию деформации в кристаллах PMN-32PT, в которых однодоменная орторомбическая фаза в поле [011] возникала в полях, меньших $2.2 \mathrm{kV} / \mathrm{cm}$.

На рис. 3, $a, b$ представлены временные зависимости оптических, акустических и диэлектрических свойств в поле вдоль [111] в кристаллах РТ29. Так же, как и в двух предыдущих случаях, индуцированный фазовый переход происходит с временной задержкой, так как в образце имеются доли областей как релаксорной стеклоподобной, так и ромбоэдрической фазы, которые начинают поворачиваться в направлении приложения поля. Так как наибольшее число этих PNR имеет локальную ромбоэдрическую симметрию, их вектор спонтанной поляризации ориентирован вдоль направления [111] [17]. 
Однако в отсутствие поля спонтанная поляризация PNR пространственно распределена вдоль восьми направлений [111], что приводит к многодоменному состоянию образца и нулевой поляризации. Как видно из рисунка, наибольшее изменение всех трех изучаемых свойств в электрическом поле происходит в одном и том же временном интервале, что свидетельствует о переходе образца в монодоменную ромбоэдрическую фазу. Увеличение оптического пропускания после фазового перехода служит подтверждением этого. Предположение о монодоменизации образца согласуется с данными для электрического поля, приложенного вдоль [011] (рис. 2, a,b). В отличие от поля, приложенного вдоль [011], в случае поля [111] индуцируется ромбоэдрическая монодоменная фаза.

Полученные нами результаты согласуются с данными работы [18], посвященной изучению диэлектрических и пьезоэлектрических свойств в ряде кристаллов $\mathrm{PMN}-x \mathrm{PT}$, расположенных вблизи МФГ, при приложении электрических полей разного направления. В ней было показано, что приложение электрического поля вдоль оси спонтанной поляризации [111] индуцирует монодоменное состояние образца, при этом диэлектрические и пьезоэлектрические восприимчивости кристаллов очень низкие, так же, как и в нашем эксперименте (см. рис. 3, a, кривая 3 ).

\section{4. Заключение}

Таким образом, в работе проведены комплексные исследования оптических, акустических и диэлектрических свойств в ряде релаксорных твердых растворов для трех случаев приложения электрического поля. Показано:

1. При приложении электрического поля в разных направлениях после некоторого времени задержки индуцируются фазы разной симметрии, которая зависит от направления приложенного поля.

2. В небольших полях $\sim 2 \mathrm{~V} / \mathrm{cm}$ при приложении поля вдоль [011] и [111] индуцируются монодоменные фазы ромбическая и ромбоэдрическая соответственно. Подтверждением этого являются максимальные изменения всех трех исследуемых характеристик в одном и том же временном интервале, а также совпадение временных зависимостей оптического пропускания на разных длинах волн света.

3. В отличие от полей, направленных вдоль [011] и [111], приложение поля вдоль [001] индуцирует многодоменную моноклинную фазу, о чем свидетельствует как несовпадение по времени аномалий акустических и диэлектрических свойств с оптическими свойствами, так и разные времена, требуемые для поляризации областей разных размеров $(1.3$ и $0.63 \mu \mathrm{m})$.

4. Только комплексные одновременные измерения всех трех свойств позволяют сделать вывод о индуцировании монодоменной или многодоменной фазы.

\section{Список литературы}

[1] S.B. Vakhrushev, J.M. Kiat, B. Dkhil. Solid State Commun. 103, 477 (1997).

[2] E.V. Colla, E.Y. Koroleva, N.M. Okuneva, S.B. Vakhrushev. Phys. Rev. Lett. 74, 1681 (1995).

[3] E.V. Colla, M.B. Weissman. Phys. Rev. B 72, 104106 (2005).

[4] E.V. Colla, D. Vigil, J. Timmerwilke, M.B. Weissman. Phys. Rev. B 75, 214201 (2007).

[5] E.V. Colla, N. Jurik, Y. Liu, M.E.X. Delgado, M.B. Weissman, D.D. Vieland, Z.-G. Ye. J. Appl. Phys. 113, 184104 (2013).

[6] Л.С. Камзина, Л.А. Кулакова, Н. Luo. ФТТ 56, 9, 1809 (2014).

[7] Л.С. Камзина, Л.А. Кулакова. ФТТ 58, 1, 177 (2016).

[8] Л.С. Камзина, Л.А. Кулакова. ФТТ 57, 11, 2164 (2015).

[9] Л.С. Камзина, Л.А. Кулакова. ФТТ 59, 2, 290 (2017).

[10] Л.С. Камзина, Л.А. Кулакова. ФТТ 59, 10, 1945 (2017).

[11] X. Liu, S. Zhang, J. Luo, T.R. Shrout, W. Cao. Appl. Phys. Lett. 97, 032902 (2011).

[12] T.H. Kim, S. Kojima, J.-H. Ko. J. Appl. Phys. 111, 054103 (2012).

[13] H. Luo, G. Xu, H. Xu, P. Wagn. Jpn. J. Appl. Phys. 39, 5581 (2000).

[14] S.J. Zhang, L. Lebrun, S. Rhee, C.A. Randall, T.R. Shrout. Appl. Phys. Lett. 81, 892 (2002).

[15] M. Roth, E. Mojaev, E. Dul'kin, P. Gemeiner, B. Dkhil. Phys. Rev. Lett. 98, 265701 (2007).

[16] M. Shanthi, L.C. Lim. J. Appl. Phys. 106, 114116 (2009).

[17] H. Cao, J. Li, D. Vieland, G. Xu. Phys. Rev. B 73, 184110 (2006).

[18] Z. Feng, X. Zhao, H. Luo. J. Appl. Phys. 100, 024104 (2006) 